\title{
Primary branchiogenic carcinoma in the first branchial cleft of a child
}

\author{
YAOSHU TENG ${ }^{1}$, HAIFEI WANG ${ }^{1}$, RUJUN XU $^{2}$, XINMU WANG ${ }^{3}$, XIAOLIN CAO ${ }^{1}$, YU FANG $^{1}$ and JIN ZHU $^{1}$ \\ Departments of ${ }^{1}$ Otorhinolaryngology, ${ }^{2}$ Pathology, and ${ }^{3}$ Stomatology, \\ Hangzhou First People's Hospital, Hangzhou 310006, P.R. China
}

Received April 25, 2012; Accepted August 13, 2012

DOI: $10.3892 / \mathrm{ol} .2012 .912$

\begin{abstract}
To our knowledge, no case report of first branchial cleft carcinoma has been described in children. Herein, we report a case of squamous cell carcinoma that developed in the vestigium of the first branchial apparatus in a 10-year-old girl who had suffered from first branchial cleft sinus since childhood. Following a wide surgical excision and neck dissection, the patient has remained in good health for 2.5 years without developing any primary or recurrent tumors locally or elsewhere. Based on this case report, it is significant to check whether a patient has ever suffered from congenital lateral cervical sinus, fistulae or cysts during a history consultation, as this could be employed, in addition to the criteria of Khafif et al, to aid the diagnosis of primary branchiogenic carcinoma.
\end{abstract}

\section{Introduction}

Primary branchiogenic carcinoma arises from the vestigium of the branchial apparatus and is exceedingly rare. Since Von Volkmann originally described this unique entity in $1882(1,2)$, there has been skepticism and controversy over issues concerning its existence and diagnosis. After reviewing 250 cases in 1950, Martin et al (3) revealed that the previous diagnostic criteria for this carcinoma were inaccurate and questionable, and thus proposed four stringent criteria to establish an accurate diagnosis of primary branchiogenic carcinoma, which were later modified by Khafif et al (4). Studies have revealed that most of the so-called primary branchial cleft cyst carcinomas are actually cystic cervical metastases resulting from oropharyngeal carcinoma, most commonly originating in the tonsils in the upper aerodigestive tract (5). To date, there are only approximately 40 cases of primary branchiogenic carcinoma reported in the literature,

Correspondence to: Dr Jin Zhu, Department of Otorhinolaryngology, Hangzhou First People's Hospital, 261 Huansha Road, Hangzhou 310006, P.R. China

E-mail: zhujinhyh@yahoo.com.cn

Key words: branchial cleft cyst, branchiogenic carcinoma, metastasis, occult primary, diagnosis which were found mostly in men aged 40-60 with the majority of cases involving second branchial cleft cysts $(6,7)$. In terms of primary carcinoma in the first branchial vestigium, to the best of our knowledge, there are only 5 reported cases, all of which occurred in adults $(1,8,9)$. Herein, we report the first case of primary first branchial cleft carcinoma in a 10 -year-old Chinese girl who had suffered from first branchial cleft sinus from childhood. The unique clinical and histological features of the patient meet the criteria established by Khafif et al (4) for the diagnosis of this rare carcinoma. The patient's mother provided informed consent. In this report, we discuss the clinical observations, diagnosis and therapeutic options of the case.

\section{Case report}

A 10-year-old girl presented at her local hospital in July 2009 with a 2 -week history of a painful, progressively enlarging mass in the right postauricular area. Physical examination revealed a large $(3.5 \times 3.5 \mathrm{~cm})$ well-defined soft tissue mass. The mass was fluctuant, slightly painful and tender, with an aperture that drained a small amount of purulent fluid. An aperture in this area was first noted by the patient's parents when she was 2 years old. The patient was diagnosed with an infected branchial cleft sinus and treated with an intravenous antibiotic for 4 days; however, this further aggravated the pain. The mass was incised and a large amount of thick, white, sticky pus was drained. A small amount of slightly bloody discharge from the incision site continued over the following two months. Granulation tissue grew at the site to a size of approximately $2 \times 2 \mathrm{~cm}$.

The patient was transferred to Hangzhou First People's Hospital, Hangzhou for further evaluation and treatment. When the patient arrived at our hospital, no signs of otorrhea, hearing loss, odynophagia or dysphagia were noted. Needle probing detected a sinus ostium-like structure, $1.2 \mathrm{~cm}$ in depth, behind the right ear. The sinus tract opening was surrounded by granulation tissue approximately $2 \times 2 \mathrm{~cm}$ in size (Fig. 1A). The skin was slightly red and swollen and the patient felt slight pain when the area was gently pressed. The skin of the right external acoustic meatus remained intact. An examination of the oropharynx revealed grade II enlarged tonsils on both sides without ulceration. Examinations by fiber optic nasopharyngoscopy and laryngoscopy revealed no abnormalities. 

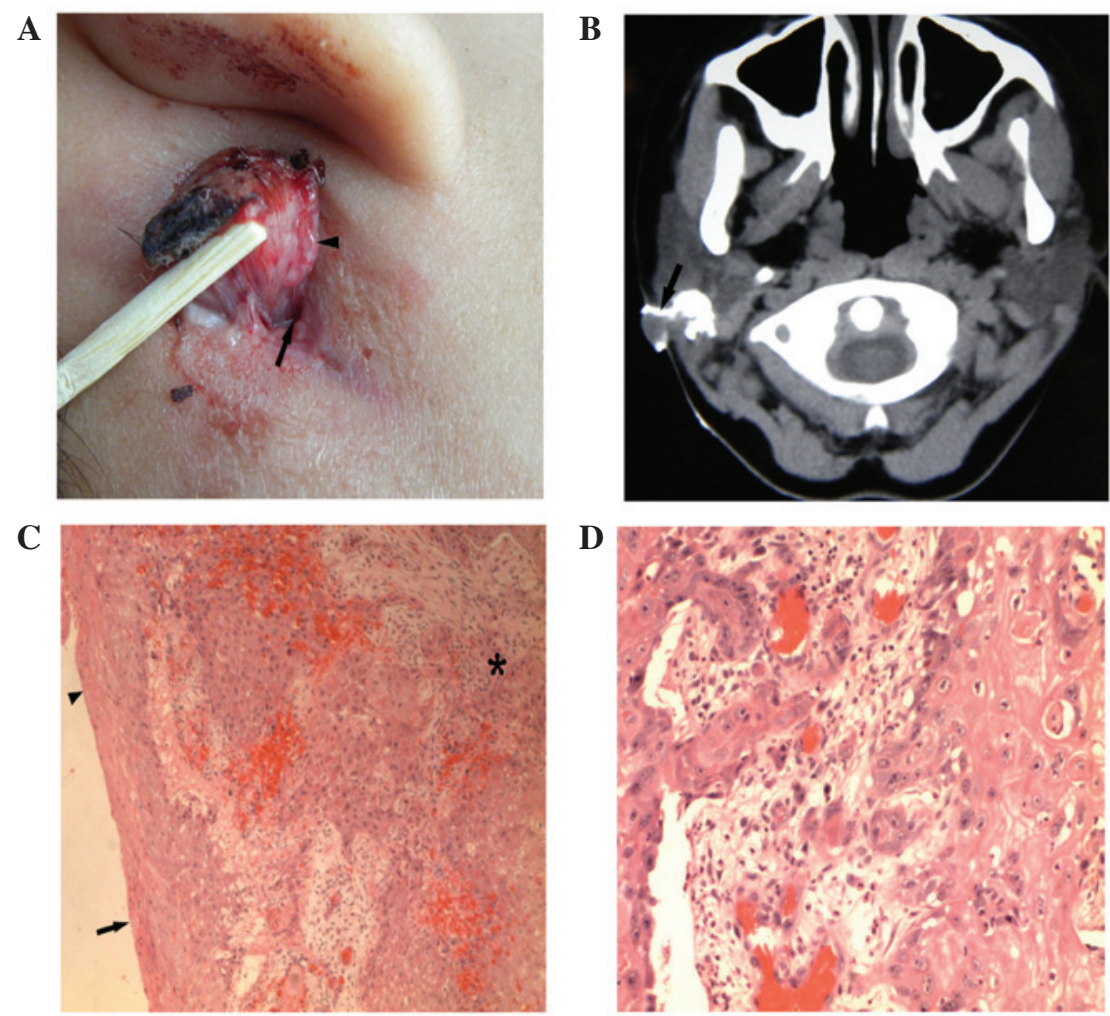

Figure 1. (A) A macrograph of the sinus ostium-like structure. The sinus tract opening (arrow) is visible on the skin behind the right ear with newly formed granulation tissue (arrowhead). (B) A CT scan of irregular soft tissue behind the right ear following meglumine diatrizoate injection. The accumulation of the contrast medium is visible in the sinus tract (arrow). (C) A micrograph shows that the sinus tract in the area of dysplasia (arrow) is partially lined with normal stratified squamous epithelium (arrowhead). A focal area of invasive squamous cell carcinoma (asterisk) is observed (hematoxylin and eosin stain, original magnification $\mathrm{x} 40$ ). (D) A higher magnification of the carcinomatous area shows well-differentiated invasive squamous cell carcinoma (hematoxylin and eosin stain, original magnification $\mathrm{x} 100)$.

A contrast-enhanced computed tomography (CT) scan of the neck revealed irregular soft tissue, which was approximately $2.4 \times 1.7 \mathrm{~cm}$ in size, and a $3 \mathrm{~cm}$ long sinus tract in the right postauricular area. The accumulation of meglumine diatrizoate was visible in the sinus tract and revealed a cystic structure with an unclear boundary at the end of the tract, reaching the parotid gland (Fig. 1B). However, a full CT scan of the head and neck revealed the absence of any identifiable primary malignant tumor and no obvious swelling in the upper neck lymph nodes.

The patient received surgery following the injection of methylene blue into the sinus ostium to act as a marker. A $3 \mathrm{~cm}$ fusiform incision was made around the granulation tissue. During surgery, the sinus tract and the cystic structure were carefully separated from the peripheral tissues. It was noted that the wall of the sinus tract was incomplete and adhered to the front border of the upper sternocleidomastoid muscle. The cystic portion had reached the bifurcation of the facial nerve trunk and the parotid gland, and slightly adhered to the gland. Histopathological examination of the tissue samples obtained from the surgery revealed normal stratified squamous epithelium on the majority of the internal surface of the sinus tract; however, an atypical disturbed epithelium structure was revealed in certain areas. In particular, invasive squamous cell carcinoma was observed next to the dysplastic epithelium (Fig. 1C and D). These findings indicate a transition from normal squamous epithelium to carcinoma. Ten days later, the patient underwent extensive surgical resection in the area of the right parotid gland. The submandibular gland was also removed; however, the facial nerve was separated and remained intact. Histopathological examination of the resected specimens revealed no residual tumor in the right parotid gland and no tumor cells in the lymph nodes in the neck above the hyoid bone. The patient had no evidence of tumor recurrence during the 2.5-year postoperative follow-up.

\section{Discussion}

The term 'primary branchiogenic carcinoma' refers to the carcinomatous degeneration of the normal epidermal lining to form congenital embryonal vestigial remnants of the branchial apparatus. Its causes are not definitively known but may include irradiation and chronic inflammation. Current documentation on branchial cleft carcinoma is mostly in the form of case reports and retrospective analyses (5).

Due to their shared anatomic region, primary branchial cleft cyst carcinoma needs to be distinguished from cystic cervical metastases arising from occult primary tumors in the upper aerodigestive tract, which have a much higher incidence rate $(1,2,5,7,8)$. The majority of cystic cervical metastases originate from Waldeyer's ring, including the nasopharynx, tonsil and the base of the tongue. The incidence of such cystic degeneration has been reported to be 33 to $50 \%$ (2). Through histological comparisons of branchial cleft cysts and normal 
lymph nodes, Krogdahl found that primary branchiogenic tissue could have lymphoid elements, such as follicles with germinal centers but lacked three characteristics of lymph nodes: peripheral lobulation, internodular trabeculae and perinodal sinuses. The diagnosis of primary branchiogenic carcinoma may be eliminated if the lesion has these three characteristics (10).

Based on an analysis of 250 cases, Martin et al (3) proposed four criteria for the diagnosis of primary branchiogenic carcinoma in 1950: i) the location of the tumor in the anatomic region of the branchial cleft cyst or sinus, i.e. along the line anterior to the sternocleidomastoid muscle between the tragus and the clavicle; ii) a histological appearance of the tumor consistent with its origin from the branchial vestiges, i.e., squamous cell carcinoma; iii) an absence of the occurrence of other primary tumors within a 5-year follow-up period following diagnosis; and iv) the histological demonstration of a cancer developing in the wall of an epithelial-lined cyst situated in the lateral site of the neck. Since some of the criteria, particularly the third point, although important in theory, are not quite feasible in clinical practice, Khafif et al (4) modified the criteria to emphasize the significance of clinical and histopathological evidence. These criteria are widely acknowledged by the majority of scholars, including Martin et al. Furthermore, they all agree that histopathological evidence is the most crucial criterion, i.e., histopathological proof of gradual transition of normal squamous epithelium into invasive carcinoma $(1,11,12)$. Clinically, it is also important that there is an absence of any identifiable primary malignant tumors following a thorough evaluation of the patient with endoscopy (nasopharyngoscopy, laryngoscopy, bronchoscopy and esophagoscopy) and radiographic examinations.

In the present case, histopathologically, the transition of normal squamous epithelium into invasive carcinoma was observed in the sinus tract situated in the region of the first branchial apparatus. Fiber optic nasopharyngoscopy, laryngoscopy and a full CT scan revealed no other abnormalities. Hence, the patient was diagnosed as having a primary first branchial cleft carcinoma, which is consistent with the criteria Khafif et al proposed. This patient also had a significant medical history which included an aperture of the first branchial cleft sinus found when the patient was 2 years old. This led to a carcinomatous degeneration of the normal epidermal lining in the subsequent years. Therefore, we propose another criterion for confirming the diagnosis of primary branchiogenic carcinoma: a clinical history of congenital lateral cervical sinus, fistulae or cysts and the development of squamous cell carcinoma. This may supplement the criteria of Khafif et al.

The treatment for primary branchiogenic carcinoma involves surgery and radiation therapy. Extensive surgical excision and neck dissection are often recommended. Adjuvant radiation therapy is helpful in preventing a relapse, particularly for patients who receive limited resection, are unwilling to undergo further lymphatic dissection or have positive lymph nodes $(5,13)$. The patient in the present case received a wide surgical excision of the lesion in addition to the dissection of the lymph nodes of the upper neck. Since no tumor cells were found in the lymph nodes, radiotherapy was not performed. The outcome of the treatment appears to be satisfactory, as indicated by a 2.5 -year postoperative follow-up.

\section{References}

1. Bhanote M and Yang GC: Malignant first branchial cleft cysts presented as submandibular abscesses in fine-needle aspiration: report of three cases and review of literature. Diagn Cytopathol 36: 876-881, 2008.

2. Briggs RD, Pou AM and Schnadig VJ: Cystic metastasis versus branchial cleft carcinoma: a diagnostic challenge. Laryngoscope 112: 1010-1014, 2002.

3. Martin H, Morfit HM and Ehrlich H: The case for branchiogenic cancer (malignant branchioma). Ann Surg 132: 867-887, 1950.

4. Khafif RA, Prichep R and Minkowitz S: Primary branchiogenic carcinoma. Head Neck 11: 153-163, 1989.

5. Jereczek-Fossa BA, Casadio C, Jassem J, et al: Branchiogenic carcinoma - conceptual or true clinico-pathological entity? Cancer Treat Rev 31: 106-114, 2005.

6. Maturo SC, Michaelson PG and Faulkner JA: Primary branchiogenic carcinoma: the confusion continues. Am J Otolaryngol 28: 25-27, 2007.

7. Girvigian MR, Rechdouni AK, Zeger GD, Segall H, Rice DH and Petrovich Z: Squamous cell carcinoma arising in a second branchial cleft cyst. Am J Clin Oncol 27: 96-100, 2004.

8. Roche JP, Younes MN, Funkhouser WK and Weissler MC: Branchiogenic carcinoma of a first branchial cleft cyst. Otolaryngol Head Neck Surg 143: 167-168, 2010.

9. Park SS and Karmody CS: The first branchial cleft carcinoma. Arch Otolaryngol Head Neck Surg 118: 969-971, 1992.

10. Krogdahl AS: Carcinoma occurring in branchial cleft cysts. Acta Otolaryngol 88: 289-295, 1979.

11. Lin YC, Fang SY and Huang RH: Branchiogenic squamous cell carcinoma: a case report. Int J Oral Maxillofac Surg 33: 209-212, 2004.

12. Hong KH, Moon WS and Chung GH: Radiological appearance of primary branchial cleft cyst carcinoma. J Laryngol Otol 113: 1031-1033, 1999.

13. Singh B, Balwally AN, Sundaram K, Har-El G and Krgin B: Branchial cleft cyst carcinoma: myth or reality? Ann Otol Rhinol Laryngol 107: 519-524, 1998 